\title{
ANALYSE POLLINIQUE DES MIELS DES ÎLES DE L'OCÉAN INDIEN
}

\author{
Pollen anAlysis OF hONeys fROM the INDIAN OCEAN ISLANDS
}

ANÁlisiS POlíNICO DE MIELES PROCEDENTES DE LAS ISLAS DEL OCÉANO INDICO

\author{
T.M. Rasoloarijao ${ }^{1}$ Z. Ramamonjisoa Ralalaharisoa ${ }^{1 *}$ P. Ramavovololona $^{1}$ V. Porphyre ${ }^{2}$
}

Mots-clés : Miel - Provenance géographique - Palynologie Litchi sinensis - Adansonia spp. - Eucalyptus spp. - Réunion Madagascar - Rodrigues.

Keywords: Honey - Geographical provenance - Palynology Litchi sinensis - Adansonia spp. - Eucalyptus spp. - Réunion Madagascar - Rodrigues.

Palabras clave: Miel - Procedencia geográfica - Palinología Litchi sinensis - Adansonia spp. - Eucalyptus spp. - Reunión Madagascar - Rodrígues.

I es îles de l'océan Indien, lieux sensibles de biodiversité floLristique, produisent différents types de miels, dont les miels de litchi, d'eucalyptus, de baobab, de tamarin et de mille fleurs. Les appellations courantes sont données par habitude à partir des observations de butinage, de couleur et autres caractères. Pour l'intégration dans le circuit commercial national ou international, il est indispensable de pouvoir différentier les productions et de leur attribuer des appellations conformes aux données scientifiques. L'analyse pollinique ou mélissopalynologie, basée sur la relation entre les grains de pollen contenus dans le miel et la plante productrice de nectar, est la méthode qui permet à la fois de vérifier une appellation florale et son origine géographique (1). Le but de ce travail a été de caractériser les miels des différentes îles selon leur origine botanique et leur provenance géographique, et de déterminer leur profil pollinique.

Cinquante-neuf échantillons de miels, dont 20 en provenance de Madagascar, 31 de Rodrigues et 8 de la Réunion ont été analysés. Les analyses ont consisté à identifier, dénombrer les taxons polliniques et déterminer les types de miel suivant les fréquences relatives du pollen dominant des différents miels. Les taxons polliniques associés au pollen dominant de chaque type de miel ont été relevés pour chaque région.

Deux principaux types de miel - miels de litchi et d'eucalyptus ont été reconnus à travers 123 taxons polliniques rencontrés dans l'ensemble des échantillons étudiés pour lesquels 70 p. 100 se sont révélés conformes aux appellations données, attestées par la présence d'un pollen dominant (fréquence $\geq 45$ p. 100). Ce sont des miels monofloraux (2). La figure 1 montre les champs microscopiques des principaux types de miels monofloraux analysés et leur diagramme pollinique : les miels de litchi et d'eucalyptus.

La classification ascendante hiérarchique des résultats de I'analyse pollinique (figure 2) a permis de faire des regroupements par rapport à l'origine botanique et aux régions de production. Les miels de litchi de Madagascar étaient associés aux pollens de Macaranga spp., de Mimosa pudica et d'Elaeis guineensis, alors que ceux de la Réunion étaient caractérisés par la présence de pollens de Casuarina spp. et de Schinus spp. Chez les miels d'eucalyptus de Rodrigues, la fréquence relative du pollen d'eucalyptus a été supérieure à 65 p. 100. Les miels dits de baobab de Madagascar n'étaient pas monofloraux mais étaient caractérisés par la présence de pollens d'Adansonia spp. et par la prédominance des pollens de Sorghum vulgare et de Ziziphus mauritiana.

Les résultats obtenus ont permis de vérifier l'origine florale des miels étudiés et de les caractériser du point de vue de leur origine géographique. Toutefois, I'authentification des miels monofloraux mérite d'être complétée par des analyses physicochimiques et sensorielles; il conviendrait aussi d'augmenter le nombre d'échantillons par type de miel et par pays pour une meilleure représentativité des résultats. Des inventaires dans les îles de l'océan Indien aideraient à découvrir d'autres types de miels monofloraux.

\section{BIBLIOGRAPHIE}

1. LOUVEAUX J., MAURIZIO A., VORWOHL C., 1970. Commission internationale de botanique apicole de I'U.I.S.B. Les méthodes de la mélissopalynologie. Apidologie, 1 : 211-227.

2. RASOLOARIJAO T., 2012. Analyses polliniques des miels de Madagascar et de deux îles des Mascareignes (île de la Réunion - île Rodrigues). Mém. DEA, Fac. Sci., Univ. Antananarivo, Madagascar, 80 p.

Accepted 30 April 2015; Online publication June 2015

1. Laboratoire de palynologie, faculté des sciences, Antananarivo, Madagascar.

2. Cirad, UMR Selmet, station de Ligne-Paradis, 7 chemin de l'IRAT, 97410 Saint-Pierre, Réunion, France.

* Auteur pour la correspondance

E-mail : joari.ramamonjisoa@gmail.com 

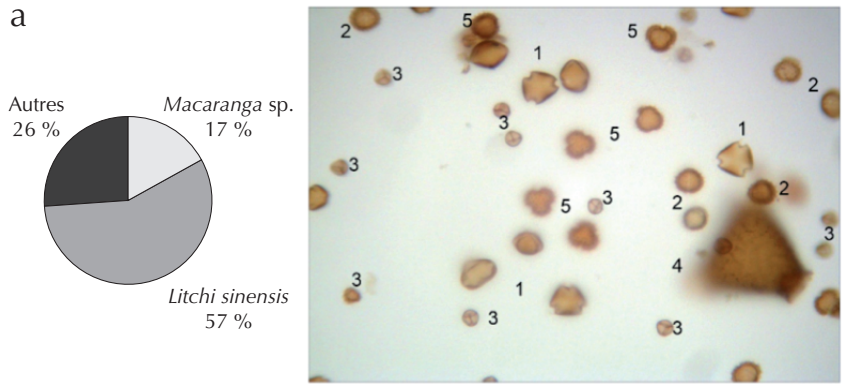

1 : Litchi sinensis ; 2 : Macaranga sp. ;

3 : Mimosa sp. ; 4 : Grevillea robusta ;

5 : Psiadia altissima

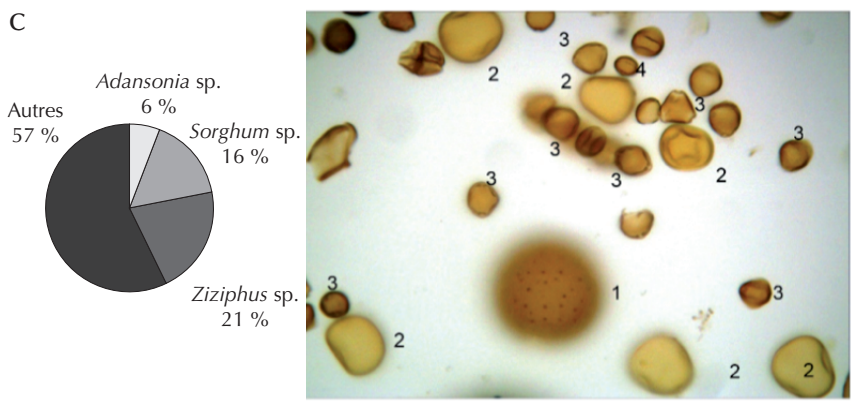

1 : Adansonia za ; 2 : Sorghum vulgare ;

3 : Ziziphus mauritiana; 4 : Dalbergia sp.

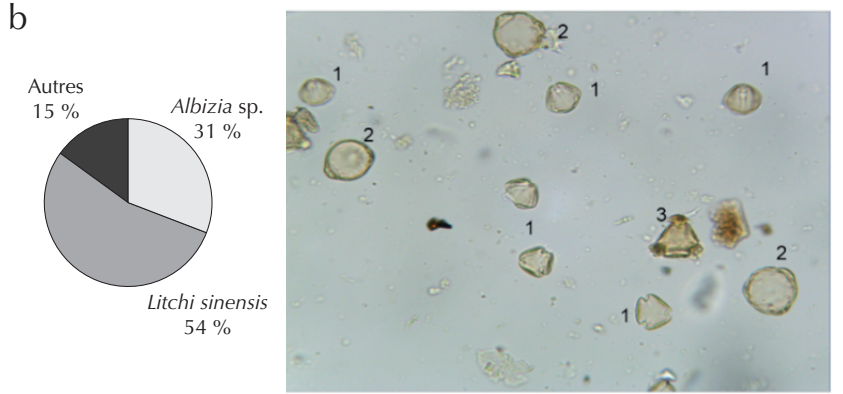

1 : Litchi sinensis ; 2 : Casuarina sp. ;

3 : Eucalyptus sp.
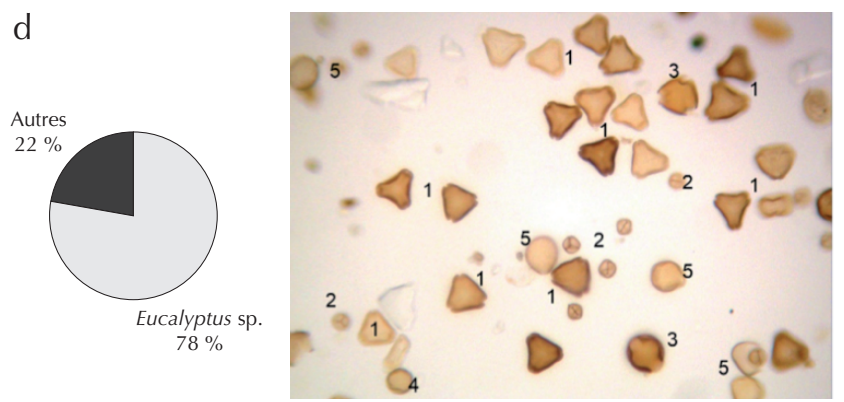

1 : Eucalyptus sp. ; 2 : Mimosa sp. ;

3 : Carissa sp. ; 4 : Leucaena sp. ; 5 : Poaceae

Figure 1 : champs microscopiques des principaux types de miels monofloraux analysés et leur diagramme pollinique : (a) miel de litchi de Madagascar ; (b) miel de litchi de la Réunion ; (c) miel de baobab de Madagascar ; (d) miel d'eucalyptus de Rodrigues.
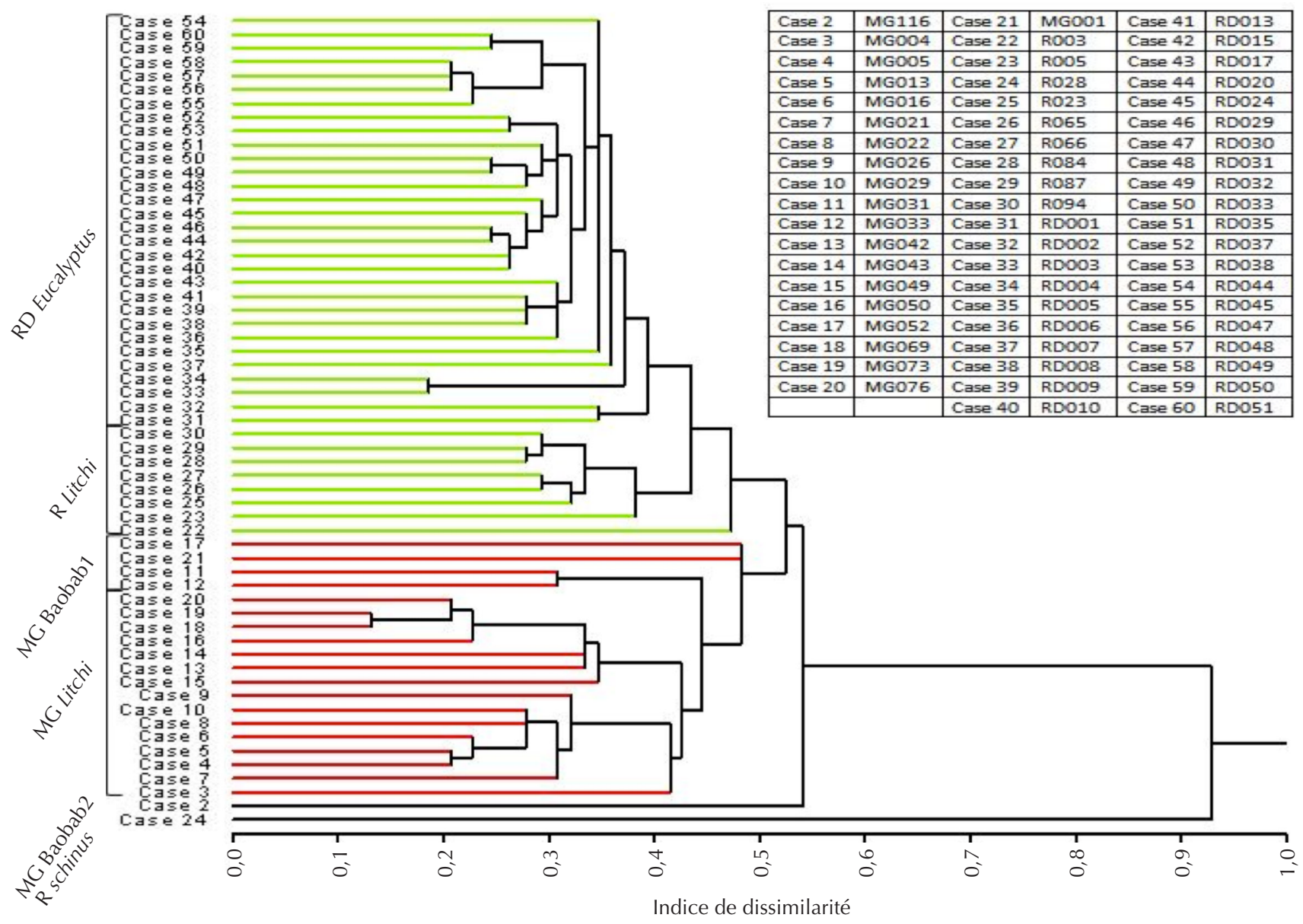

Figure 2 : classification ascendante hiérarchique des miels étudiés ; MG : Madagascar ; $R$ : Réunion ; RD : Rodrigues (Rasoloarijao, 2012). 